\title{
Resistencia al cambio y trabajo por turnos en trabajadores de la banca
}

\author{
Valero R., Daniel V.* \\ Castilla Ramos, Orquídea**
}

\section{Resumen}

Este artículo muestra los factores de resistencia al cambio ante la implementación de un esquema de trabajo por turnos para el personal del departamento de bases de datos de una institución bancaria venezolana, con el objetivo de diseñar un plan para el manejo del cambio, a través del cual la gerencia pueda intervenir y lograr superar dicha resistencia. La investigación consistió en un estudio de caso, que parte de realizar un diagnóstico que permitió identificar factores de resistencia como la disminución del ingreso económico, amenaza a hábitos familiares e intereses propios y seguridad personal; para luego diseñar un plan de manejo del cambio que le permitirá a la gerencia del área implantar el esquema de horarios de trabajo por turnos, con el menor impacto posible sobre el personal.

Palabras clave: Cambio organizacional, resistencia al cambio, turnos de trabajo, jornada laboral, trabajadores bancarios.

\section{Resistence to Change and Shift Work Among Bank Workers}

\begin{abstract}
This article shows resistance to change factors in the light of implementing a new scheme for shift work among personnel from the data processing department in a Venezuelan banking institution, with the objective of designing a plan to handle change, through which management can intervene and overcome the aforementioned resistance. The research was a case study that begins with performing a diagnostic that made it possible to identify resistance factors, such as diminished income, a threat to family habits, personal interests and personal security, and then design a plant to handle

Recibido: 11-01-11. Aceptado: 17-07-11

* Postgrado en Desarrollo Organizacional. Universidad Simón Bolívar. Caracas-Venezuela.

E-mail: dvaleror@gmail.com

** Profesor en el Departamento de Ciencia y Tecnología del Comportamiento. Universidad Simón Bolívar. Caracas-Venezuela. E-mail: ocastilla@usb.ve
\end{abstract}


change that will allow management in the area to implant the scheme of work schedules by shift with the least impact on personnel.

Key words: Organizational change, resistance to change, work shifts, workday, bank workers.

\section{Introducción}

Las organizaciones buscan constantemente mecanismos para hacer un mejor uso de su capacidad instalada y disminuir la cantidad de tiempo ocioso que puede generarse en los procesos. Por esta razón, algunas empresas debido a su naturaleza, tratan de establecer canales que permitan a sus clientes tener acceso a los servicios que proveen, la mayor cantidad de horas al día y la mayor cantidad de días del año; otras para responder a regulaciones gubernamentales, como es el caso de las instituciones bancarias en Venezuela, deben garantizar el acceso a sus servicios las veinticuatro (24) horas del día.

Para ofrecer la disponibilidad de los servicios las instituciones bancarias en Venezuela no sólo requieren una logística adecuada y una plataforma tecnológica moderna y estable, sino contar con personal que posea la experiencia necesaria para solventar cualquier problema que se pueda presentar en dicha plataforma en el menor tiempo posible, disminuyendo los tiempos en que los servicios de la organización no están disponibles o están disponibles pero con una degradación importante en su calidad.

Uno de los mecanismos implementados para garantizar la atención de incidentes en la plataforma, es establecer guardias de trabajo no presenciales que permiten contar con personal dedicado a solucionar problemas cuando sea reque- rido, a través de accesos remotos a la organización o trasladándose a las instalaciones si es preciso. Esta modalidad, implementada en la institución bancaria en estudio, no siempre ha garantizado la oportuna atención de los problemas, lo que ha llevado a modificar el horario de trabajo actual de su personal del departamento de base de datos e implantar un esquema de dos turnos que permita contar con éste en el lugar de trabajo durante 16 horas al día.

El hecho de alterar el horario de trabajo corresponde a un cambio organizacional, y autores como Böhrt (2001), Hodge et al. (2003) y Kotter y Schlesinger (2008) plantean que ante cualquier cambio propuesto se pueden presentar reacciones adversas por parte de las personas que se verán afectadas por dicho cambio, por lo que es necesario diseñar estrategias que permitan mitigarlas o evitarlas completamente, así como reforzar los comportamientos que apoyan e impulsan la ejecución del cambio.

En este sentido, el propósito general de este artículo es presentar un plan para enfrentar la resistencia al cambio, que permita implementar a la gerencia un esquema de trabajo por turnos para el personal del departamento de base de datos de la gerencia de almacenamiento en una institución bancaria del país. Se realizó un estudio de caso, que implicó el diagnóstico de la situación actual de este grupo de trabajadores, a fin de determinar la existencia de resistencia al cambio 
Resistencia al cambio y trabajo por turnos en trabajadores de la banca

Valero R., Daniel V. y Castilla Ramos, Orquídea

ante el nuevo sistema de turnos por parte de los miembros del departamento y contribuir a identificar los factores específicos de resistencia presentes en el personal, así como algunas alternativas para superar esa situación propuestas por ellos. Luego se diseñó un plan que permitirá a la gerencia del área implementar el esquema de horarios de trabajo propuesto, con el menor impacto posible sobre el personal del departamento de bases de datos, a través de la mitigación de los factores de resistencia encontrados durante el diagnóstico.

\section{El trabajo por turnos}

De acuerdo a Thierry y Meiman (1994, citado por Buxton, 2003) y Suárez (1999), el trabajo por turnos se define como aquel que es desarrollado por distintos grupos consecutivos, cumpliendo cada uno de ellos una jornada laboral, donde cada grupo generalmente lleva a cabo el mismo trabajo de forma que la operación se realice entre 16 y 24 horas, más tiempo del permitido para un solo trabajador. La jornada laboral corresponde al tiempo que cada trabajador dedica a la ejecución del trabajo para el cual ha sido contratado. En Venezuela, la Ley Orgánica del Trabajo (Congreso Nacional, 1997) en su artículo 189 la define como:

“... el tiempo durante el cual el trabajador está a disposición del patrono y no puede disponer libremente de su actividad y de sus movimientos. Se considera que el trabajador está a disposición del patrono desde el momento en que llega al lugar donde deba efectuar su trabajo, o donde deba recibir órdenes o instrucciones respecto al trabajo que se ha de efectuar en cada día, hasta que pueda disponer libremente de su tiempo y de su actividad."

En cuanto a su duración, dicha Ley establece en su artículo 195 los límites que rigen la materia, indicando que:

“...la jornada diurna no podrá exceder de ocho (8) horas diarias, ni de cuarenta y cuatro (44) semanales; la jornada nocturna no podrá exceder de siete (7) horas diarias, ni de cuarenta (40) semanales; y la jornada mixta no podrá exceder de siete y media $(71 / 2)$ horas por día, ni de cuarenta y dos (42) por semana. Se considera como jornada diurna la cumplida entre las 5:00 a.m. y las 7:00 p.m. Se considera como jornada nocturna la cumplida entre las 7:00 p.m. y las 5:00 a.m. Se considera como jornada mixta la que comprende períodos de trabajo diurnos y nocturnos. Cuando la jornada mixta tenga un período nocturno mayor de cuatro (4) horas, se considerará como jornada nocturna."

Asimismo, el trabajo por turnos también puede ser definido como el arreglo de los horarios de trabajo que utilizan dos o más grupos (turnos) de trabajadores, de forma de extender la operación del ambiente de trabajo más allá de las horas de oficina convencionales (Pati et al., 2001). Cada turno realiza las mismas actividades y tiene las mismas responsabilidades, para que las operaciones puedan continuar más allá de lo que permite un solo trabajador (Buxton, 2003). Esta es la definición que más se ajustaría al caso de estudio de este artículo.

Algunas definiciones, tales como la de Bird (2007), establecen que se puede 
considerar trabajo por turnos a cualquier actividad laboral realizada fuera del horario entre 07:00AM y 07:00PM, de lunes a viernes, aunque las horas específicas pueden variar de acuerdo a la legislación de cada país. Sin embargo, la secuencia de los turnos usualmente sigue un patrón predecible y regular, lo cual no puede ser confundido con horarios de trabajo irregulares en las que las horas de trabajo varían de forma irregular e impredecible (Thierry y Meijman, 1994; citado por Buxton, 2003).

En la actualidad, un esquema de horarios convencional se considera de cuarenta (40) horas a la semana, ocho (8) horas al día de lunes a viernes, pero con la introducción del trabajo por turnos, la semana laboral de cinco (5) días puede cambiar a una semana laboral de tres (3) o cuatro (4) días, de acuerdo a la duración establecida para el turno (Occupational Health Clinics for Ontario Workers Inc., 2005). En Venezuela, La Ley Orgánica del Trabajo establece en su artículo 201 que el trabajador por turnos puede exceder los límites diarios establecidos en el referido artículo 195, siempre y cuando el total de horas trabajadas en un periodo de ocho semanas no exceda tales límites.

El trabajo por turnos puede tener diferentes diseños. El Cuadro 1 lo resume.

Diversas razones llevan a las organizaciones a la implementación de esquemas de turnos. Estas pueden ser económicas, de servicio o técnicas (mencionadas por Mellor, 1986; Suarez, 1999; Rosa y Colligan, 1997; Occupational Health Clinics for Ontario Workers Inc., 2005). En el caso particular de la banca, servicios tales como banca telefónica, cajeros electrónicos, puntos de venta, banca por Internet, dispensadores automáticos de servicio y equipos de autoservicio entre otros, que si bien han existido desde hace varios años, actualmente están concebidos para permitir a sus clientes realizar diversas operaciones durante las 24 horas del día, y constituyen por lo tanto un ejemplo claro de la razón de servicio.

Autores como Finn (1981:32), han descrito algunas de las ventajas del trabajo por turnos:

- Es una posibilidad de empleo para quienes no logran obtener un trabajo diurno.

- Brinda la oportunidad de mantener un segundo trabajo, y así aumentar los ingresos.

- Estudiantes que requieren costear sus estudios y que el trabajo no genere conflicto con sus horarios de clases.

- Los turnos de la tarde y la noche permiten contar con mayor tiempo durante el día para actividades personales, y según el esquema de rotación acumular varios días libres consecutivos.

- Podría ser una forma de escape a responsabilidades y/o situaciones familiares indeseadas.

- Contar con un lugar de trabajo más relajado, por menor supervisión y menos interrupciones por parte del personal gerencial.

A pesar de esto, generalmente, trabajar de esta manera es dañino, con consecuencias tales como trastornos del sueño, digestivos y nerviosos e interferencia con las relaciones de pareja, familiares y sociales, así como accidentes laborales o fuera del trabajo (Mellor, 
Resistencia al cambio y trabajo por turnos en trabajadores de la banca Valero R., Daniel V. y Castilla Ramos, Orquídea

\section{Cuadro 1} Diseños del trabajo por turnos

\begin{tabular}{|c|c|c|}
\hline Autores & Criterio de diseño & Tipos y características \\
\hline $\begin{array}{l}\text { Nogareda y Nogareda } \\
\qquad(1997)\end{array}$ & Continuidad de las operaciones & $\begin{array}{l}\text { Sistema continuo: la operación se realiza de for- } \\
\text { ma ininterrumpida, durante todo el día y durante } \\
\text { todos los días del año, suponiendo más de tres } \\
\text { turnos y trabajo nocturno. } \\
\text { Sistema discontinuo: la operación es interrum- } \\
\text { pida durante la noche y fines de semana, supo- } \\
\text { niendo dos turnos, uno de mañana y uno de tarde. } \\
\text { Sistema semi-continuo: la operación se realiza } \\
\text { durante las veinticuatro (24) horas del día, con in- } \\
\text { terrupciones durante los fines de semana, supo- } \\
\text { niendo tres turnos, mañana, tarde y noche. }\end{array}$ \\
\hline Carcelén (2000) & $\begin{array}{l}\text { Forma en que se asignan } \\
\text { los trabajadores al turno } \\
\text { (o permanencia del } \\
\text { trabajador en el turno). }\end{array}$ & $\begin{array}{l}\text { Estático o cerrado: los trabajadores son asigna- } \\
\text { dos de forma permanente a un horario particular. } \\
\text { Abierto o rotatorio: los trabajadores son asigna- } \\
\text { dos a turnos diferentes de forma periódica. } \\
\text { Mixto: un grupo de trabajadores son asignados a } \\
\text { un turno fijo y otro grupo de trabajadores son asig- } \\
\text { nados a turnos rotatorios. }\end{array}$ \\
\hline $\begin{array}{l}\text { Hill (2002, citado por } \\
\text { Steinmetz, 2007) }\end{array}$ & $\begin{array}{l}\text { Dirección y velocidad } \\
\text { de rotación. }\end{array}$ & $\begin{array}{l}\text { Hacia delante o hacia atrás: establece la direc- } \\
\text { ción, es decir si la asignación se realiza desde tur- } \\
\text { nos con horarios matutinos a turnos con horarios } \\
\text { nocturnos, o viceversa. } \\
\text { Rápida o lenta: establece qué tan rápido se cam- } \\
\text { bia la asignación del trabajador al turno. La veloci- } \\
\text { dad puede ser rápida y el trabajador permanecer } \\
\text { uno o dos dias en un turno antes de ser cambiado } \\
\text { a otro, o puede ser lenta y permanecer una o va- } \\
\text { rias semanas en el turno antes de ser cambiado a } \\
\text { otro. }\end{array}$ \\
\hline
\end{tabular}

Fuente: Elaboración propia (2010).

1986). Estas dependen del esquema utilizado (cerrado, rotatorio o mixto), así como de la dirección y velocidad de rotación, y pueden ser de diferentes tipos (Buxton, 2003; Bird, 2007; Pati et al., 2001; Finn, 1981; Folkard et al., 2005). Independientemente de las razones que tenga una organización, el hecho de alterar el esquema de horarios de trabajo corresponde a un cambio organizacional, y por lo tanto, es necesario hacer referencia a este concepto.

\section{El cambio organizacional}

Las organizaciones son sistemas abiertos, deben identificar las amenazas y oportunidades de su entorno así como responder a las nuevas condiciones planteadas, lo cual puede requerir cambios para adaptarse y sobrevivir (Hogde et al., 2003; Robbins, 2004). Factores como nuevas regulaciones, aumento de la competencia, aparición de productos y/o servicios sustitutos y desarrollos tecnológicos, 
las obligan a ejecutar pequeños cambios por lo menos una vez cada año y cambios mayores cada cuatro o cinco años (Kotter y Schlesinger, 2008). El actor fundamental en este proceso es el agente de cambio, individuo o grupo que dirige las actividades de cambio; un consultor contratado para ayudar a la organización o un integrante de la misma sin importar su nivel jerárquico (Robbins, 2004).

Según Hodge et al. (2003), un proceso de cambio planificado se inicia con el reconocimiento de la necesidad, identificada por una evaluación de las condiciones internas y externas de la organización y el reconocimiento de una diferencia entre las condiciones actuales y las deseadas, lo que crea la motivación para el cambio. Seguidamente, los directores evalúan cuáles cambios son necesarios y la velocidad con que deben llevarse a cabo; un consultor externo podría evitar evaluaciones sesgadas y soluciones influidas por la cultura actual de la organización. Después se indica la forma en que será dirigido el cambio, a través de la fase de planificación y análisis; se definen metas de cambio cuantitativas y verificables, para que la evaluación futura del éxito del cambio pueda llevarse a cabo. En este punto se establecen las estrategias de cambio y se procede a su ejecución, para lo cual es necesario que los miembros de la organización modifiquen o eliminen determinadas conductas. Luego, la evaluación de los resultados determinará en qué medida los objetivos fueron alcanzados y si es necesario realizar ajustes a las estrategias de cambio o desarrollar nuevas. El proceso finaliza con el recongelamiento de las conductas para que se conviertan en un componente permanente de la cultura de la organización.

Estos mismos autores (Hodge et al., 2003) indican que es posible introducir cambios en las metas y estrategia de la organización, en las personas, productos y servicios, tecnología, cultura y en su estructura y diseño. Hacen hincapié en que una modificación en una de las áreas, seguramente lo requerirá en alguna o varias de las otras. Durante un cambio en el diseño del trabajo y al definir las tareas que deben realizarse así como los resultados que se esperan de ellas, es necesario determinar el momento en que deben realizarse; es posible encontrar que se requiera contar con trabajadores durante más o menos horas que antes y/o pueden variar los días de la semana en que son requeridos, trayendo como consecuencia la modificación de los turnos de trabajo actuales para un conjunto de actividades en particular.

De acuerdo a Böhrt (2001), es necesario prever las reacciones ante el cambio, así como sus posibles consecuencias, independientemente de lo irracionales que puedan parecer, para establecer estrategias que permitan mitigarlas o evitarlas completamente. Según este mismo autor, al modificar las condiciones laborales, los procedimientos, el espacio de trabajo, o cualquier otro componente del diseño del trabajo (como el horario o turno de trabajo), las personas muestran un comportamiento de resistencia.

\section{La resistencia al cambio}

Puede definirse como la reacción natural de los individuos y los sistemas sociales originada de la necesidad por 
Resistencia al cambio y trabajo por turnos en trabajadores de la banca

Valero R., Daniel V. y Castilla Ramos, Orquídea

una situación relativamente estable (Bennebroek, 2003). Algunos estudiosos le han dado a la resistencia una connotación negativa, describiéndola como un conjunto de fuerzas que obstruyen los esfuerzos de los líderes; sin embargo, otros han descrito su lado positivo y la consideran una fuente importante de información que permite ejecutar el cambio de forma exitosa (Szabla, 2007).

La resistencia puede ser abierta e inmediata. Sin embargo, la forma más peligrosa es la implícita o encubierta, en la cual los individuos que no se sienten cómodos con la situación solicitan traslados, renuncian, descuidan el trabajo reduciendo de forma evidente la calidad del resultado, incrementan su ausentismo, pierden la motivación y la lealtad hacia la organización (Robbins, 2004).

Sobre sus causas, en el Cuadro 2 se resumen las relacionadas con el individuo y las organizacionales. La resistencia al cambio puede manejarse y mitigar sus causas. Kotter y Schlesinger (2008:130), proponen seis tácticas para que los agentes de cambio manejen la resistencia:

- Educación y comunicación: comunicar las ideas en discusiones personales, memorandos, presentaciones a grupos, los cuales permiten que los individuos afectados por el cambio entiendan la necesidad de éste y su lógica. Es muy útil cuando la resistencia es por la falta de información; sin embargo, requiere que el agente de cambio tenga la confianza de aquellos que se resisten.

- Participación e inclusión: involucrar a quienes se pueden ver afectados en el diseño y la implementación del cam- bio; es necesario que el agente de cambio escuche a los demás e incluya sus ideas y sugerencias en el plan de cambio, de forma que se sientan comprometidos con el éxito del proceso. Requiere mucho tiempo para involucrar a todas las personas y no puede aplicarse a cambios que deben realizarse de forma inmediata. Es posible que la solución que se obtenga sea pobre, si no se maneja el proceso cuidadosamente.

- Facilitación y apoyo: si se percibe miedo y angustia en los miembros de la organización, la capacitación les permitirá adquirir nuevos conocimientos y habilidades. También se puede premiar al individuo con tiempo libre luego de un período de gran demanda. Su principal desventaja es que requiere gran cantidad de tiempo e inversión económica y aún así no se garantiza el éxito.

- Negociación y acuerdo: consiste en ofrecer incentivos a quienes se resisten al cambio de forma activa o pasiva. Es muy útil cuando se determina que alguien va a perder algo de valor como resultado del cambio, pero debido a su poder en la organización puede ser un factor de resistencia determinante.

- Manipulación y cooptación: es el uso selectivo de la información, se oculta parte de ella o se crean rumores falsos, influyendo en la estructuración de eventos. La cooptación consiste en dar a un individuo, o al líder de un grupo, un rol en el diseño del cambio de forma de obtener su apoyo, aún cuando realmente no se desea su consejo. Aunque esta táctica puede ser menos 


\section{Cuadro 2}

\section{Causas de la resistencia al cambio}

\begin{tabular}{|c|c|c|}
\hline $\begin{array}{l}\text { Causa/ Fuente } \\
\text { de Resistencia }\end{array}$ & $\begin{array}{c}\text { Causa } \\
\text { Especifica }\end{array}$ & Caracteristicas \\
\hline \multirow[t]{7}{*}{$\begin{array}{l}\text { DEL INDIVIDUO } \\
\text { Robbins (2004) y Kotter } \\
\text { y Schlesinger (2008) }\end{array}$} & Hábitos & $\begin{array}{l}\text { Los seres humanos crean hábitos o respuestas programadas } \\
\text { para enfrentar situaciones que viven de forma frecuente. Cuan- } \\
\text { do un cambio toma lugar, la inclinación a responder de la forma } \\
\text { acostumbrada da origen a una resistencia. }\end{array}$ \\
\hline & $\begin{array}{c}\text { Malentendidos } \\
\text { o falta de confianza }\end{array}$ & $\begin{array}{l}\text { Cuando las personas no conocen o no entienden las implicacio- } \\
\text { nes del cambio, pueden percibir que la pérdida será mayor a la } \\
\text { ganancia y resistir el cambio. Esta situación se presenta común- } \\
\text { mente cuando no existe confianza entre el agente de cambio y } \\
\text { quienes se verán afectados por el. }\end{array}$ \\
\hline & $\begin{array}{l}\text { Miedo a lo } \\
\text { desconocido }\end{array}$ & $\begin{array}{l}\text { Las personas resisten el cambio cuando creen que no serán ca- } \\
\text { paces de desarrollar las habilidades y conductas que serán re- } \\
\text { queridas. }\end{array}$ \\
\hline & $\begin{array}{c}\text { Factores } \\
\text { económicos }\end{array}$ & $\begin{array}{l}\text { Está muy ligado con el miedo a lo desconocido ya que en situa- } \\
\text { ciones en las que el ingreso de la persona depende de su pro- } \\
\text { ductividad, los cambios en las tareas o rutinas pueden causar } \\
\text { preocupación de tipo económico si la persona no cree ser capaz } \\
\text { de ejecutar las nuevas tareas de forma adecuada. }\end{array}$ \\
\hline & $\begin{array}{l}\text { Baja tolerancia } \\
\text { al cambio }\end{array}$ & $\begin{array}{l}\text { Los seres humanos tienen una habilidad de cambio limitada y } \\
\text { varía de persona en persona. Las organizaciones pueden re- } \\
\text { querir cambios de forma inesperada y rápidamente y algunas } \\
\text { personas son incapaces de cambiar sus actitudes y conductas a } \\
\text { la velocidad que requieren sus organizaciones. Esto puede lle- } \\
\text { var a un individuo a resistir un cambio aún cuando entiende que } \\
\text { puede ser beneficioso a nivel personal y organizacional. }\end{array}$ \\
\hline & $\begin{array}{l}\text { Seguridad e } \\
\text { intereses } \\
\text { propios }\end{array}$ & $\begin{array}{l}\text { Si una persona percibe que perderá algo de valor como resulta- } \\
\text { do del cambio, se enfocará en sus propios intereses y no en los } \\
\text { de la organización, y defenderá lo que considera valioso enfren- } \\
\text { tándose al cambio. Algunas personas se resisten al cambio } \\
\text { para proteger su imagen personal, ya que consideran que cam- } \\
\text { biar es admitir que sus decisiones o creencias pasadas eran } \\
\text { erróneas. }\end{array}$ \\
\hline & $\begin{array}{l}\text { Procesamiento } \\
\text { selectivo de la } \\
\text { información }\end{array}$ & $\begin{array}{l}\text { Los individuos tienen una percepción del mundo y procesarán la } \\
\text { información ignorando aquello que contradice sus percepcio- } \\
\text { nes de forma que estas queden intactas. }\end{array}$ \\
\hline \multirow[t]{3}{*}{$\begin{array}{l}\text { DE LA ORGANIZACIÓN } \\
\text { Robbins (2004) y } \\
\text { Hannan y Freeman } \\
\text { (1984, citado por } \\
\text { Otero, 2002) }\end{array}$} & $\begin{array}{c}\text { Inercia } \\
\text { organizacional }\end{array}$ & $\begin{array}{l}\text { Causada por mecanismos y estructuras formales dentro de las } \\
\text { organizaciones que buscan estabilidad [Robbins (2004)]. La } \\
\text { inercia es alta cuando la velocidad de reorganización de las es- } \\
\text { tructuras es menor a la velocidad con que cambian las condicio- } \\
\text { nes del entorno [Hannan y Freeman (1984, c.p. Otero, 2002)] }\end{array}$ \\
\hline & Inercia grupal & $\begin{array}{l}\text { Originada por las regulaciones y reglas (formales e informales), } \\
\text { restringe el cambio aún cuando los individuos que conforman el } \\
\text { grupo deseen cambiar }\end{array}$ \\
\hline & $\begin{array}{l}\text { Alcance que se } \\
\text { da al cambio }\end{array}$ & $\begin{array}{l}\text { Si es aplicado sólo a un subsistema tenderá a ser anulado por } \\
\text { los sistemas mayores. }\end{array}$ \\
\hline
\end{tabular}


Resistencia al cambio y trabajo por turnos en trabajadores de la banca

Valero R., Daniel V. y Castilla Ramos, Orquídea

\section{Cuadro 2. Causas de la resistencia al cambio (Continuación)}

\begin{tabular}{ccc}
$\begin{array}{c}\text { Causa/ Fuente } \\
\text { de Resistencia }\end{array}$ & $\begin{array}{c}\text { Causa } \\
\text { Especifica }\end{array}$ & Caracteristicas \\
\hline & $\begin{array}{c}\text { Amenaza a la } \\
\text { experiencia de } \\
\text { los grupos } \\
\text { especializados }\end{array}$ & $\begin{array}{c}\text { Es la que perciben los individuos ante un cambio de los patrones } \\
\text { de la organización. La modificación de procesos de toma de de- } \\
\text { cisiones implica redistribución del poder, que puede ser consi- } \\
\text { derada como desafío a las relaciones establecidas existentes. }\end{array}$ \\
$\begin{array}{c}\text { Amenaza percibida } \\
\text { por distribución } \\
\text { de recursos }\end{array}$ & $\begin{array}{l}\text { La amenaza percibida por quienes se benefician de la forma ac- } \\
\text { tual en que se distribuyen los recursos, ya que el cambio puede } \\
\text { afectar las distribuciones futuras }\end{array}$ \\
\hline
\end{tabular}

Fuente: Elaboración propia (2010).

costosa que las otras, es necesario tener cuidado para que aquellos que son objetos de la cooptación no descubran que están siendo engañados, ya que se puede obtener una respuesta desfavorable que aumente aún más la resistencia.

- Coerción explícita e implícita: consiste en obligar, implícita o explícitamente, a que se acepte el cambio a través de amenazas (despido, transferencias, o pérdida de beneficios). Requiere que el agente de cambio tenga un poder considerable y es peligrosa porque la gente se resentirá ante los cambios forzados; sin embargo, sería la única opción cuando el cambio sea impopular sin importar la forma en que se maneje.

Estas tácticas pueden y deben ser combinadas de acuerdo a la situación que se enfrenta para obtener resultados positivos, y es un error común que los agentes de cambio utilicen siempre la misma táctica independientemente de la situación que se presenta.

Para manejar la resistencia al cambio adecuadamente en un grupo organizacional primero hay que conocer si existe o no García (2005), presenta una he- rramienta o instrumento para el diagnóstico del nivel de resistencia al cambio, que considera tanto el aspecto técnico como el aspecto humano del mismo, y utiliza como premisa la teoría de campos de fuerza de Kurt Lewin. Plantea la necesidad de realizar mediciones en función de las personas involucradas o afectadas por el cambio, tales como los individuos o grupos con poder para sancionar o legitimar los procesos de cambio, los agentes de cambio y quienes tienen que incorporar a sus prácticas los nuevos procesos, métodos o herramientas definidos como resultado del proceso de cambio (blancos de cambio), siempre evaluando los factores de resistencia ligados al individuo y a la organización.

Según García (2009), la herramienta está concebida para ser aplicada a muestras grandes, pero puede servir de guía para la elaboración de una herramienta que permita determinar el nivel de resistencia al cambio en grupos pequeños de individuos, por lo que es aplicable en casos como el investigado.

Sobre la resistencia al cambio de horario o a trabajar por turnos, Nogareda y Nogareda (1997) en un estudio realizado en enfermeras que trabajan bajo esta 
modalidad, indican que los aspectos relacionados con la organización del trabajo deben ser considerados durante una evaluación que busca encontrar las posibles causas de resistencia a la modificación del horario de trabajos, de un esquema regular a un esquema por turnos, principalmente cuando los afectados tengan experiencia en esta forma de trabajo, satisfactoria o no, que puede influir en el caso que está siendo estudiado.

Así mismo, Nogareda y Nogareda (1998) presentan algunos criterios que permiten evaluar el trabajo por turnos, en cuanto a factores tales como variables asociadas a la falta de sueño, problemas asociados a la salud física y mental, vida familiar y social, factores de personalidad y contexto laboral. Aunque este último trabajo está enfocado en la evaluación de un esquema por turnos en funcionamiento, es posible estudiar la percepción de los empleados sobre estas variables ante la propuesta de implantación de un esquema de horarios por turnos, ya que puede apoyar la búsqueda de las causas de una posible resistencia al cambio.

\section{Trabajo por turnos en trabajadores de base de datos bancarias: un estudio de caso}

La organización objeto de estudio es un instituto bancario de capital $100 \%$ venezolano que se encuentra entre los primeros diez lugares en el ranking de Venezuela de bancos comerciales y universales (Medina y Borgucci, 2005). La gerencia de esta institución desea que los incidentes que se presenten, y que afectan el ciclo de la producción, tengan un efecto mínimo y sean resueltos dentro del tiempo meta definido. Para cumplir con este objetivo de forma exitosa propuso la implementación de un esquema de trabajo por turnos, para los departamentos que actualmente laboran en la jornada ordinaria definida para el personal administrativo y técnico, la cual consiste en un único turno de 08:00AM a 04:30PM de lunes a viernes. La propuesta consistió en la sustitución del único turno actual por dos turnos que permitan disponer de personal en el lugar de trabajo desde las 06:00AM hasta las 10:00PM de lunes a viernes, mediante la siguiente jornada: un turno de 06:00 AM a 02:00 PM y el otro de 02:00 a 10:00 PM.

La propuesta requería implementarse inicialmente en el departamento de base de datos, encargado de garantizar la integridad, confidencialidad y disponibilidad de los datos almacenados en los diferentes sistemas de gestión de bases de datos de la organización. Una vez planteada, los gerentes superiores al departamento de bases de datos, percibieron incomodidad e incertidumbre en el personal del mismo en cuanto a abandonar el esquema actual de horarios de trabajo.

El estudio de caso persiguió en primer lugar, determinar la existencia de la resistencia percibida al nuevo esquema por turnos así como sus causas, y en segundo lugar, a partir del diagnóstico realizado, elaborar un plan de manejo del cambio, para facilitar la implementación del esquema de trabajo por turnos en el departamento de bases de datos, que tomara en cuenta las necesidades detectadas y sirviera de apoyo a la gerencia del banco.

El diagnóstico de la situación actual implicó reuniones previas con la gerencia inmediata superior del grupo de trabajo, 
Resistencia al cambio y trabajo por turnos en trabajadores de la banca

Valero R., Daniel V. y Castilla Ramos, Orquídea

con el objeto de conocer la retroalimentación recibida por parte del personal en cuanto a la propuesta de implementación de turnos de trabajo y, en particular, identificar los aspectos que llevan al personal supervisor a considerar que existe resistencia al cambio en este personal.

La información suministrada en dichas reuniones permitió diseñar un guión o guía para una entrevista estructurada (de acuerdo a lo establecido por Kerlinger y Lee, 2002) con veintidós (22) preguntas, principalmente reactivos o preguntas de tipo abierto y otras de alternativa fija. Algunas buscaban evaluar los factores de resistencia descritos por García (2005) y otro grupo la percepción de los miembros del departamento hacia los factores descritos por Nogareda y Nogareda (1997; 1998). Finalmente, se incorporaron preguntas para evaluar los aspectos mencionados por los gerentes en las reuniones previas. Esta guía de entrevista fue revisada por tres jueces expertos en diseño de instrumentos.

La entrevista fue realizada a los doce (12) miembros del departamento en las instalaciones de la empresa; no se hizo en los puestos de trabajo para evitar que fueran interrumpidos por solicitudes de trabajo. La mayor parte de los trabajadores entrevistados tiene edades comprendidas entre los 30 y 34 años o entre 45 y 49 años (64\%), son casados (75\%), de sexo masculino (75\%), con hijos $(67 \%)$, sólo en edad escolar (33\%) o cursando secundaria (25\%). La mayoría de estos empleados $(67 \%)$ ha prestado servicio en la organización y se ha mantenido en el cargo actual durante menos de dos años y medio. El resto tiene más de cuatro años en la organización o en el cargo, y los de mayor antigüedad sólo seis años. Están distribuidos igualitariamente para trabajar en las tres plataformas de bases de datos de la organización (cuatro en cada una).

Los resultados encontrados en cada una de las categorías identificadas al analizar las respuestas dadas a la entrevista y los datos demográficos registrados durante el diagnóstico, se dispusieron en tablas de frecuencia y porcentaje. Con la finalidad de identificar los factores de resistencia o variables de los entrevistados con diferencias estadísticamente significativas, se aplicó la prueba no paramétrica Chi-cuadrado $\left(\chi^{2}\right)$, que según Siegel y Castellan (1995), es el tipo de prueba adecuada cuando el tamaño de la muestra es pequeño y se aplica a datos clasificatorios (categorías) medidos en escala nominal, condiciones que se presentan en este estudio. Se muestran sólo aquellas variables en donde se encontraron asociaciones estadísticamente significativas según el resultado de la prueba.

Entre los hallazgos relevantes del diagnóstico, vale destacar:

- Los entrevistados indican que no fueron consultados al momento de definir las bandas horarias de los turnos, ni participaron en el diseño del esquema de turnos propuesto. Así mismo, opinan que la organización no ha considerado posibles efectos de su implementación, como la dificultad para coordinar compartir puestos de trabajo, imposibilidad de garantizar presencia del personal en los dos turnos (por vacaciones o enfermedad, ya que la institución no ampliará la plan- 
tilla de doce trabajadores) comunicación como equipo de trabajo, servicios de alimentación y seguridad del personal en horas en las que no trabaja más personal ni se dispone de esos servicios.

- La mayoría (83\%) considera que el nuevo esquema de turnos propuesto debe ser modificado, sobre todo en lo de compartir puestos de trabajo y equipos, ya que alguna actividad que se esté realizando en un turno no podría ser continuada por el empleado que se incorpora al siguiente turno, ya que implicaría entrega de contraseñas y otros datos de usuario de red, lo cual es contraindicado por seguridad de información.

- La totalidad indicó que no se han planteado recompensas al personal para aceptar trabajar en uno de los turnos propuestos y tampoco las consideran necesarias, ya que debe ser una decisión personal si las condiciones solicitadas por cada uno de ellos están dadas.

- La mayoría (más del 50\%) considera que su trabajo no se verá impactado por la implementación del esquema de turnos planteados. Cuatro (4) entrevistados consideran que el esquema de trabajo por turnos propuesto impactará negativamente su trabajo si trabajan en el turno de $2 \mathrm{pm}$ a $10 \mathrm{pm}$, debido al "trabajo solitario" o "asignación de trabajo de menor dificultad".

- Los entrevistados trabajan sobretiempo de forma regular (después de las 4:30 pm) y consideran que el esquema de turnos incidiría en la cantidad de sobretiempo trabajado, cau- sando una disminución del número de horas trabajadas producto de jornadas adicionales, ya que como personal en sitio en un turno cubrirán parte del horario que actualmente cumplen como personal de guardia, por lo que sus intereses personales se verán afectados negativamente.

- $67 \%$ considera que su rutina familiar se verá afectada de forma negativa en caso de trabajar en alguno de los turnos propuestos.

- $83 \%$ afirma que sólo trabajar en el turno de $2 \mathrm{pm}$ a $10 \mathrm{pm}$ afectaría de forma negativa su vida social, ya que prácticamente eliminaría la posibilidad de recreación de lunes a viernes.

- El 83\% considera que trabajar en cualquiera de los turnos pudiese ser favorable para ellos ya que permitiría "reducción del sobretiempo realizado" o "mayor tiempo libre por trabajar fuera de horas pico". Otros aspectos mencionados fueron: "continuar estudios" de post-grado, "realizar otra actividad productiva" y "pasar más tiempo con su familia e hijos".

- Los aspectos necesarios señalados para aceptar trabajar en uno de los turnos propuestos fueron disponibilidad de estacionamiento y/o transporte y alimentación. La mayoría indicó que para el turno nocturno, el puesto de estacionamiento debe ser asignado en el mismo edificio de la sede principal, por razones de seguridad personal y de los equipos de computación y telefónicos asignados durante el turno. Otra opción sería garantizar el transporte Hogar-Empresa (para el turno de 6am a 2pm) y Em- 
Resistencia al cambio y trabajo por turnos en trabajadores de la banca

Valero R., Daniel V. y Castilla Ramos, Orquídea

presa-Hogar (para el turno de 2pm a 10pm).

- La mitad está dispuesta a trabajar en alguno de los turnos propuestos. Adicionalmente, ninguno considera conveniente implantar turnos rotativos ni desea trabajar bajo ese esquema. De los que sí están dispuestos, cuatro (4) prefieren el turno de $6 \mathrm{am}$ a $2 \mathrm{pm}$, dos (2) de ellos de la plataforma SQL Server (aplicación para el manejo de base de datos de la empresa Microsoft), uno (1) de la plataforma ORACLE (aplicación para el manejo de base de datos de la empresa Oracle) y uno (1) de la plataforma DB2 (aplicación para el manejo de base de datos de la empresa IBM). Los otros (2) empleados prefieren el turno de $2 \mathrm{pm}$ a $10 \mathrm{pm}$, uno (1) de ellos de la plataforma SQL Server y el otro de la plataforma ORACLE.

La prueba Chi-cuadrado determinó la asociación estadísticamente significativa sólo entre las variables estado civil y afectación de la rutina y/o vida familiar, plataforma con la que se trabaja y afectación de intereses personales, presencia de hijos y afectación de la rutina y/o vida familiar, y entre tener hijos y disposición para trabajar en alguno de los turnos propuestos. Cabe acotar que la muestra era muy homogénea, por lo cual en varios casos no se pudo realizar el cálculo del coeficiente de asociación $\left(\chi^{2}\right)$.

En la Tabla 1 se observa la relación entre el estado civil y la afectación de la vida y/o rutina familiar. Los entrevistados solteros consideran que no se verán afectados por los turnos propuestos. De los nueve (9) entrevistados casados, ocho (8) piensan que la implantación del esquema de turnos afectará su vida familiar.

Para estas variables la probabilidad asociada al coeficiente Chi-cuadrado fue de 0,005 por debajo del valor crítico establecido $p=a l f a=0,05$ (ver Tabla 2), lo cual señala la existencia de diferencias estadísticamente significativas entre la percepción de los solteros y casados, en cuanto a la forma en la cual el esquema de turnos propuesto afectará su vida y/o rutina familiar. Específicamente los solteros expresan que no los afectará negativamente mientras que los casados sí.

La relación entre el tipo de plataforma con la que se trabaja y la afectación o no de los intereses personales se observa en la Tabla 3. Los miembros del equipo de la plataforma SQL Server y los del equipo de plataforma DB2 consideran que sus intereses personales pueden verse afectados negativamente. En con-

Tabla 1

Relación estado civil con afectación de la rutina y/o vida familiar

\begin{tabular}{rccc}
\hline & \multicolumn{3}{c}{ ¿Cómo afectará su rutina y/o vida familiar? } \\
\cline { 2 - 4 } & Negativamente & No les afecta & Total \\
\hline ESTADO CIVIL Soltero & 0 & 3 & 3 \\
ESTADO CIVIL Casado & 8 & 1 & 9 \\
Total & 8 & 4 & 12 \\
\hline
\end{tabular}

Fuente: Elaboración propia con base en entrevistas realizadas (septiembre, 2009). 
Tabla 2

Chi-cuadrado para estado civil con afectación de la vida y/o intereses personales por la implantación de un nuevo esquema de turno

\begin{tabular}{|c|c|c|c|c|c|}
\hline \multicolumn{6}{|c|}{ Pruebas de chi-cuadrado } \\
\hline & Valor & $\mathrm{gl}$ & $\begin{array}{l}\text { Sig. asintótica } \\
\text { (bilateral) }\end{array}$ & $\begin{array}{l}\text { Sig. exacta } \\
\text { (bilateral) }\end{array}$ & $\begin{array}{l}\text { Sig. exacta } \\
\text { (unilateral) }\end{array}$ \\
\hline Chi-cuadrado de Pearson & $8,000^{a}$ & 1 &, 005 & & \\
\hline $\begin{array}{l}\text { Corrección por } \\
\text { continuidad }^{\mathrm{b}}\end{array}$ & 4,500 & 1 &, 034 & & \\
\hline Razón de verosimilitudes & 8,997 & 1 &, 003 & & \\
\hline $\begin{array}{l}\text { Estadístico exacto de } \\
\text { Fisher }\end{array}$ & & & &, 018 & ,018 \\
\hline $\begin{array}{l}\text { Asociación lineal por } \\
\text { lineal }\end{array}$ & 7,333 & 1 &, 007 & & \\
\hline $\mathrm{N}$ de casos válidos & 12 & & & & \\
\hline
\end{tabular}

a. 3 casillas $(75,0 \%)$ tienen una frecuencia esperada inferior a 5 . La frecuencia mínima esperada es 1,00 .

b. Calculado sólo para una tabla de $2 \times 2$.

Fuente: Elaboración propia con base en entrevistas realizadas (septiembre, 2009).

Tabla 3

Relación plataforma con afectación de los intereses personales por la implantación de un nuevo esquema de turno

\begin{tabular}{lccc}
\hline & \multicolumn{2}{c}{ Tabla de contingencia } \\
\hline & $\begin{array}{c}\text { ¿Cómo considera usted que puede verse } \\
\text { afectados sus intereses personales si se } \\
\text { implanta el esquema de turnos propuesto? }\end{array}$ & \\
\cline { 2 - 4 } & Negativamente & $\begin{array}{c}\text { Ni positivamente, } \\
\text { ni negativamente }\end{array}$ & Total \\
\hline PLATAFORMA SQL & 4 & 0 & 4 \\
PLATAFORMA ORACLE & 1 & 3 & 4 \\
PLATAFORMA DB2 & 4 & 0 & 4 \\
Total & 9 & 3 & 12 \\
\hline
\end{tabular}

Fuente: Elaboración propia con base en entrevistas realizadas (septiembre, 2009).

traste, tres (3) miembros del equipo de plataforma ORACLE piensan que no se verán afectados por este cambio, mientras que uno sólo uno (1) de ellos expresó que se verá afectado negativamente.

En la Tabla 4 se observa que la probabilidad asociada en la prueba fue de 0,018 por debajo del nivel crítico estable- cido de 0,05, lo que indica que existen diferencias significativas entre la plataforma de base de datos en la que se trabaja y considerar que se pueden ver afectados sus intereses personales con el esquema de turnos propuestos; específicamente los trabajadores de la plataforma ORACLE indicaron que no se verán afectados 
Resistencia al cambio y trabajo por turnos en trabajadores de la banca

Valero R., Daniel V. y Castilla Ramos, Orquídea

Tabla 4

Chi-cuadrado para tipo de plataforma con afectación de los intereses personales por la implantación de un nuevo esquema de turno

Pruebas de chi-cuadrado

\begin{tabular}{lrrr}
\hline \hline & Valor & gl & $\begin{array}{c}\text { Sig. asintótica } \\
\text { (bilateral) }\end{array}$ \\
\hline Chi-cuadrado de Pearson & $8,000^{\mathrm{a}}$ & 2 &, 018 \\
Razón de verosimilitudes & 8,997 & 2 &, 011 \\
$\begin{array}{l}\text { Asociación lineal por } \\
\text { lineal }\end{array}$ &, 000 & 1 & 1,000 \\
N de casos válidos & 12 & & \\
\hline \hline
\end{tabular}

a. 6 casillas $(100,0 \%)$ tienen una frecuencia esperada inferior a 5. La frecuencia mínima esperada es 1,00.

Fuente: Elaboración propia con base en entrevistas realizadas (septiembre, 2009).

positivamente ni negativamente, mientras que los de las otras dos plataformas opinaron que será negativamente. El personal de ORACLE señaló que la cantidad de trabajo que tiene en su plataforma es suficientemente alta como presencial; también piensan que tendrán un ingreso suficientemente alto a pesar de trabajar en el esquema de turnos y por lo tanto no se verán afectados negativamente por el cambio.

En la Tabla 5 se muestra la distribución de los entrevistados por presencia de hijos y afectación de la rutina y/o vida familiar. Los entrevistados con hijos manifiestan que ésta se vería afectada negativamente de implantarse el esquema de horarios, mientras que los que no tienen hijos consideran que no será afectada.

En la Tabla 6 se indica que la probabilidad asociada al coeficiente chi-cuadrado fue de 0,001 ( $p=a l f a=0,05$ ), lo cual señala la existencia de diferencias estadísticamente significativas entre la percepción de los entrevistados con hijos y sin hijos, en cuanto a la forma en la cual el esquema de turnos afectará su vida y/o rutina familiar.

La Tabla 7 muestra la distribución de los entrevistados por presencia de hijos y disposición para trabajar en alguno de los turnos propuestos. Seis (6) de los entrevistados con hijos no están dispuestos a trabajar en alguno de los turnos propuestos. Los entrevistados sin hijos están dispuestos a trabajar en alguno de los turnos propuestos.

En la Tabla 8 se observa el valor arrojado en la prueba aplicada a las variables presencia de hijos y disposición a trabajar en una de los horarios propuestos. La probabilidad asociada a esta prueba fue de 0,014 , la cual está por debajo del nivel crítico establecido de 0,05 , es decir que existen diferencias estadísticamente significativas entre los entrevistados que tienen hijos y los que no en cuanto a la disposición a trabajar en el esquema de turnos.

En conclusión, el diagnóstico permitió determinar que los factores o causas de resistencia al cambio predominan- 
Tabla 5

Relación presencia de hijos con afectación de la rutina y/o vida familiar

Tabla de contingencia

\begin{tabular}{lccc} 
& \multicolumn{2}{c}{ Tabla de contingencia } & \\
\hline & \multicolumn{2}{c}{ ¿Cómo afectará su rutina y/o vida familiar? } & \\
\cline { 2 - 3 } & Negativamente & No les afecta & Total \\
\hline TIENE HIJOS Sí & 8 & 0 & 8 \\
TIENE HIJOS No & 0 & 4 & 4 \\
Total & 8 & 4 & 12 \\
\hline
\end{tabular}

Fuente: Elaboración propia con base en entrevistas realizadas (septiembre, 2009).

Tabla 6

Chi-cuadrado para hijos con afectación de la rutina y/o vida familiar

Pruebas de chi-cuadrado

\begin{tabular}{|c|c|c|c|c|c|}
\hline & Valor & $\mathrm{gl}$ & $\begin{array}{c}\text { Sig. asintótica } \\
\text { (bilateral) }\end{array}$ & $\begin{array}{l}\text { Sig. exacta } \\
\text { (bilateral) }\end{array}$ & $\begin{array}{l}\text { Sig. exacta } \\
\text { (unilateral) }\end{array}$ \\
\hline Chi-cuadrado de Pearson & $12,000^{a}$ & 1 &, 001 & & \\
\hline $\begin{array}{l}\text { Corrección por } \\
\text { continuidad }\end{array}$ & 7,922 & 1 &, 005 & & \\
\hline Razón de verosimilitudes & 15,276 & 1 &, 000 & & \\
\hline $\begin{array}{l}\text { Estadístico exacto de } \\
\text { Fisher }\end{array}$ & & & &, 002 &, 002 \\
\hline $\begin{array}{l}\text { Asociación lineal por } \\
\text { lineal }\end{array}$ & 11,000 & 1 &, 001 & & \\
\hline $\mathrm{N}$ de casos válidos & 12 & & & & \\
\hline
\end{tabular}

a. 3 casillas $(75,0 \%)$ tienen una frecuencia esperada inferior a 5 . La frecuencia mínima esperada es 1,33 .

b. Calculado sólo para una tabla de $2 \times 2$.

Fuente: Elaboración propia con base en entrevistas realizadas (septiembre, 2009).

Tabla 7

Relación entre tener hijos con disposición para trabajar en uno de los esquemas propuestos.

\begin{tabular}{lccc}
\hline & \multicolumn{2}{c}{ Tabla de contingencia } \\
\hline & \multicolumn{2}{c}{$\begin{array}{c}\text { ¿Está usted dispuesto a trabajar en algunos } \\
\text { de los turnos propuestos? }\end{array}$} & No \\
\cline { 2 - 4 } & Sí & 6 & 8 \\
\hline TIENE HIJOS Sí & 2 & 0 & 4 \\
TIENE HIJOS No & 4 & 6 & 12 \\
\hline
\end{tabular}

Fuente: Elaboración propia con base en entrevistas realizadas (septiembre, 2009). 
Resistencia al cambio y trabajo por turnos en trabajadores de la banca

Valero R., Daniel V. y Castilla Ramos, Orquídea

Tabla 8

Chi cuadrado para hijos con disposición para trabajar en uno de los esquemas propuestos

Pruebas de chi-cuadrado

\begin{tabular}{|c|c|c|c|c|c|}
\hline & Valor & $\mathrm{gl}$ & $\begin{array}{c}\text { Sig. asintótica } \\
\text { (bilateral) }\end{array}$ & $\begin{array}{c}\text { Sig. exacta } \\
\text { (bilateral) }\end{array}$ & $\begin{array}{l}\text { Sig. exacta } \\
\text { (unilateral) }\end{array}$ \\
\hline Chi-cuadrado de Pearson & $6,000^{a}$ & 1 & ,014 & & \\
\hline $\begin{array}{l}\text { Corrección por } \\
\text { continuidad }\end{array}$ & 3,375 & 1 & ,066 & & \\
\hline Razón de verosimilitudes & 7,638 & 1 &, 006 & & \\
\hline $\begin{array}{l}\text { Estadístico exacto de } \\
\text { Fisher }\end{array}$ & & & &, 061 &, 030 \\
\hline $\begin{array}{l}\text { Asociación lineal por } \\
\text { lineal }\end{array}$ & 5,500 & 1 & 019 & & \\
\hline $\mathrm{N}$ de casos válidos & 12 & & & & \\
\hline
\end{tabular}

a. 4 casillas $(100,0 \%)$ tienen una frecuencia esperada inferior a 5. La frecuencia mínima esperada es 2,00 .

b. Calculado sólo para una tabla de $2 \times 2$.

Fuente: Elaboración propia con base en entrevistas realizadas (septiembre, 2009).

tes en este grupo son: malentendidos o falta de confianza (por no haber comunicado el cambio), factores económicos (los relacionados con no percibir ingresos por sobretiempo trabajado), seguridad e intereses propios (necesidad de servicios de transporte y alimentación, afectación de la vida o rutina familiar y social), y amenaza a la experiencia de los grupos especializados de la organización (compartir puestos de trabajo o contraseñas con compañeros de trabajo).

Para subsanar los aspectos encontrados en el diagnóstico, se propone un plan que incluye las acciones que debe realizar la gerencia de la institución bancaria con el objetivo de que conllevan a:

- Mitigar los factores de resistencia al cambio asociados a la implantación del esquema de turnos propuesto para el personal del departamento de base de datos.

- Presentar a la gerencia del área los resultados del diagnóstico efectuado.
- Recomendar soluciones a algunos de los problemas potenciales asociados a implantar el esquema de turnos, y controlar sus efectos en la ejecución de las actividades diarias; así como, opciones de modificación del esquema para facilitar la aceptación del cambio.

- Promover la aplicación de las tácticas descritas por Kotter y Schlesinger (2008) para atenuar los factores de resistencia al cambio identificados.

Este plan fue validado por la gerencia del área y recibió el visto bueno para su implementación, a continuación su estructura:

\section{Sesión No. 1}

Propósito: Plantear a la organización las posibles soluciones a las inquietudes del personal, indicando sus ventajas y desventajas, de forma que sirvan de base para las siguientes sesiones. No se considera la toma de decisiones en esta sesión. 
Objetivos: Presentar a la gerencia del área soluciones a algunos de los problemas potenciales que el personal identificó durante el diagnóstico, que podrían presentarse ante la implantación del esquema de turnos propuestos, y sus posibles consecuencias, así como propuestas para la modificación del esquema propuesto, a fin de facilitar la adopción del cambio por parte del personal.

Participantes: Consultor, Jefe de Departamento y Gerente del área.

\section{Procedimiento:}

1. En una reunión analizar las ventajas y desventajas de las posibles soluciones a las inquietudes del personal en cuanto a los servicios de alimentación, transporte y/o estacionamiento. Estas soluciones sirven de base a sesiones posteriores con el personal del Departamento.

1. 1. En cuanto a la alimentación, para las personas que tomen el turno de $2 \mathrm{pm}$ a $10 \mathrm{pm}$ (o cualquier franja que se establezca con horas nocturnas), la organización podría:

- Proveer un servicio en el lugar de trabajo.

- Cubrir los costos en los que incurra el personal por este concepto en forma de viáticos fijos o contra reembolso. Es importante tomar en consideración que, si se abandonan las instalaciones del Banco para trasladarse y adquirir alimentos, disminuye el tiempo en que se cuenta con personal en el lugar de trabajo.

1.2. Sobre el transporte y/o estacionamiento, si no se dispone de puesto de estacionamiento dentro del edificio en que laboran, la Organización podría adoptar alguna de las siguientes solucio- nes para garantizar el traslado seguro hogar-trabajo o trabajo-hogar del personal:

- Asignar un puesto de estacionamiento en el edificio.

- Proveer un servicio de transporte, dependiendo del turno en que se trabaje.

Recomendar a quienes trabajen en el turno de $2 \mathrm{pm}$ a $10 \mathrm{pm}$ el uso de algún estacionamiento público cercano y buscarlo a las 5pm, cuando se libera el acceso al estacionamiento del edificio del Banco; sin embargo, es importante tomar en cuenta, que si el personal debe abandonar las instalaciones al trasladarse para mover su vehículo de un estacionamiento cercano al Banco, disminuye el tiempo en que se cuenta con personal en el lugar de trabajo.

1.3. En relación al diseño de los turnos, se podría adoptar alguna de las siguientes propuestas:

- No establecer turnos durante todo el año, sino en periodos en que es necesario contar con personal en horario extendido, tales como: navidad, semana santa, carnavales o cualquier otro periodo en que el nivel de requerimientos, la criticidad de los proyectos o el aumento de transacciones por parte de los clientes del Banco, requieran personal en horario extendido, Esto puede facilitar que algunos empleados trabajen en los turnos propuestos al tener la seguridad de que será sólo por periodos específicos.

- Modificar la franja horaria para permitir la salida del segundo turno una o dos horas antes de las $10 \mathrm{pm}$. Este cambio significaría solapar las jornadas, por lo cual no se podrían compartir los puestos de trabajo tal y como plantea la Or- 
Resistencia al cambio y trabajo por turnos en trabajadores de la banca

Valero R., Daniel V. y Castilla Ramos, Orquídea

ganización, pero podría hacer más atractivo tomar un turno que incluya horas de la tarde y horas de la noche.

- Eliminar la restricción que establece que por cada persona que trabaje en el turno de la mañana debe haber una persona en el turno de la tarde, permitiendo así que quien desea trabajar en alguno de los nuevos turnos lo haga, ya que se aumentará la ventana de atención con personal en el lugar de trabajo, evitando por supuesto, un desbalance muy marcado en el número de personas trabajando en cada turno.

1.4. Para el aspecto familiar se puede:

- Dar prioridad al personal con hijos para el disfrute de vacaciones en periodos vacacionales escolares.

- Dar un día libre, quincenal o mensual, a los empleados que tomen el turno tarde-noche, de forma que estos dispongan de tiempo para su familia.

1.5. Durante las entrevistas preempleo presentar a los aspirantes las propuestas de horario y conocer su disposición a trabajar en estas. Si la necesidad de cubrir los turnos propuestos es alta, se deben ofrecer sólo a los aspirantes solteros sin hijos e incluir dentro del perfil de candidatos esta condición.

Duración: Esta sesión tendría una duración aproximada de ciento veinte (120) minutos.

\section{Sesión No. 2}

Propósito: Reducir los niveles de ansiedad que pueden presentarse en el personal por no conocer las razones que llevan a la Organización a plantear el cambio en los horarios de trabajo y cómo se verán impactados. Mantener o au- mentar los niveles de compromiso y lealtad de los empleados hacia la Organización, al saber que ésta ha considerado el impacto que puede tener el cambio en ellos y ha buscado la forma de beneficiar a los empleados que adopten el esquema por turnos.

Objetivos: Realizar actividades que permitan poner en práctica la táctica de "educación y comunicación" propuesta por Kotter y Schlesinger (2008), para atenuar los factores de resistencia al cambio, informando al personal perteneciente al departamento de base de datos sobre diversos aspectos del cambio propuesto y sobre el departamento.

Participantes: Consultor, miembros del Departamento, Jefe de Departamento y Gerente del área.

\section{Procedimiento:}

2.1. Comunicar y explicar:

- Los objetivos del área y, si es necesario, definir los objetivos de cada grupo de trabajo de acuerdo a la plataforma que administra.

- El rol de cada miembro del grupo en el departamento y cómo las actividades que realiza apoyan el cumplimiento de los objetivos. Si los roles no están definidos, se recomienda ejecutar una sesión adicional para realizar una intervención, aplicando la técnica de análisis de rol descrita por French y Bell (1996), con la finalidad de aclarar las expectativas del rol y las responsabilidades de cada miembro del equipo.

- La manera en que la implantación del trabajo por turnos apoyará el cumplimiento de los objetivos. Presentar las estadísticas y/o indicadores de cumplimiento de objetivos, tanto los valo- 
res actuales como los esperados. Esto permitirá que el personal no considere el cambio como un capricho.

- Los beneficios o ventajas adicionales para el empleado, como son la disminución del número de horas que el personal de guardia debe estar a disposición de la organización, y del tiempo de traslado hogar-trabajo-hogar al no realizarse en horas pico de tráfico.

- Los resultados del diagnóstico realizado, para permitir que todos manejen la misma información y cambiar la percepción del resto del equipo, haciendo que tengan una visión común de los posibles problemas que se pueden presentar.

2.2. Ya que se dispone del resultado del diagnóstico y se conocen las inquietudes y los problemas potenciales percibidos por el personal, tales como problemas de comunicación entre los turnos, dificultades percibidas al compartir el equipo y puesto de trabajo, coordinación de reuniones, transporte y alimentación, es necesario que se presenten opciones de solución a dichas situaciones y la posibilidad de que se adopte alguna de ellas. Esto permitirá evitar la sensación de improvisación por parte de la Gerencia y generará confianza en el personal.

- Primero, se mostrará a todos los miembros del equipo la información mediante una presentación digital.

- Luego, se debe permitir que los miembros del Departamento planteen sus dudas para su inmediata aclaratoria.

- En caso que no se disponga de la información necesaria para aclarar una duda, se debe establecer el compro- miso de responder a más tardar en una fecha específica y se debe respetar dicho compromiso.

Duración: La sesión tendría una duración aproximada de noventa (90) minutos, según dinámica de la reunión.

Sesión No. 3

Propósito: Involucrar a los individuos en el diseño del cambio y en la búsqueda de soluciones que permitirán la implantación exitosa del esquema de turnos propuesto, de forma que los individuos se sientan comprometidos con el éxito del proceso.

Objetivos: Realizar actividades que permitan poner en práctica la táctica de "participación e inclusión" propuesta por Kotter y Schlesinger (2008), para atenuar los factores de resistencia al cambio, al permitir que el personal de base de datos realice recomendaciones o propuestas sobre como enfrentar o mitigar los posibles problemas que ellos consideran se pueden presentar.

Participantes: Consultor, miembros del Departamento, Jefe de Departamento y Gerente del área.

\section{Procedimiento:}

3.1. A través de la intervención de Junta de Formación de Equipo del Grupo Formal, descrita por French y Bell (1996), se conducirá a los sujetos miembros del equipo a identificar sus debilidades, sus aspectos fuertes como individuos y como grupo (cómo piensan que funciona el grupo) de forma que haciendo uso de ese conocimiento y a través de la técnica de Tormenta de Ideas, realicen recomendaciones sobre cómo enfrentar o mitigar los posibles problemas que ellos consideran se pueden presentar, así como proponer cambios al esquema propuesto por la or- 
Resistencia al cambio y trabajo por turnos en trabajadores de la banca

Valero R., Daniel V. y Castilla Ramos, Orquídea

ganización, que permitirían que mayor cantidad de personas decidan cambiar su horario a uno de los turnos propuestos. Se deben tomar en cuenta aquellas propuestas realizadas durante las entrevistas, estas pueden ser presentadas al inicio de la sesión para reducir el tiempo necesario para la actividad y realizar sólo ajustes menores. Las propuestas realizadas durante las entrevistas son:

- Ajustar las franjas horarias para permitir la salida del segundo turno una o dos horas antes.

- Eliminar la restricción que establece que por cada persona que trabaje en el turno de la mañana debe haber una persona en el turno de la tarde.

Duración: Esta sesión tendrá una duración aproximada de ciento veinte (120) minutos. De ser necesario se programa la realización de una sesión adicional.

Sesión No. 4

Propósito: El propósito de estas sesiones es llegar a acuerdos entre el personal de Base de Datos y la institución, que faciliten la implantación del esquema de turnos de trabajo propuestos.

Objetivos: Realizar actividades que permitan poner en práctica la táctica de "negociación y acuerdo" propuesta por Kotter y Schlesinger (2008), descrita en el capítulo II, para atenuar los factores de resistencia al cambio, al concertar acuerdos entre las demandas realizadas por el personal de Base de Datos y la Institución sobre la implantación de un nuevo esquema de turnos de trabajo

Participantes: Consultor, miembros del departamento, Jefe de Departamento y Gerente del área.

\section{Procedimiento:}

1. Realizar sesiones individuales con cada uno de los miembros del equipo, para alcanzar acuerdos que satisfagan a ambas partes y faciliten a cada individuo el proceso de adopción de un nuevo turno de trabajo.

2. Es importante que la Organización comprenda que el personal tendrá que hacer cambios en sus rutinas y por lo tanto debe hacer concesiones en algunos aspectos, o modificar condiciones inicialmente planteadas para facilitar así el proceso de cambio.

3. Si algunos de los trabajadores solicita trabajar en alguno de los horarios propuestos de forma temporal, para vivir la experiencia y corroborar o cambiar alguna de las percepciones que tiene con respecto a trabajar en turnos, la empresa debe permitírselo ya que si la experiencia es satisfactoria aceptará más fácilmente cambiar de turno.

4. Adicionalmente, se debe llegar a acuerdos individuales para compensar la reducción esperada en los ingresos por concepto de horas extras, ya que la frecuencia de éstas pudo implicar una entrada fija de dinero para algunos trabajadores. Una forma de compensación puede ser, por ejemplo, el pago de un bono a quienes trabajen en alguno de los turnos propuestos.

Duración: La duración de las sesiones no debería superar los sesenta (60) minutos. De ser necesario se programaría la realización de una sesión adicional.

\section{Conclusiones}

El diagnóstico determinó que en el grupo estudiado existe resistencia al 
cambio ante la implementación de un esquema de trabajo por turnos y está relacionada con los factores de resistencia personales descritos por Robbins (2004) y Kotter y Schlesinger (2008).

Entre los factores de resistencia identificados se encuentran los económicos, ya que el ingreso mensual del personal se ve incrementado gracias a la ejecución de jornadas diarias de sobretiempo, que se pueden ver reducidas ante la implementación de un esquema de horarios que implique la presencia de personal en el lugar de trabajo en horario extendido. Por otra parte, el factor de desconocimiento o no comprensión de las implicaciones del cambio, que puede generar la percepción en el personal de que la pérdida será mayor a la ganancia. Se puso en evidencia la existencia de comunicación inadecuada entre el personal y la gerencia, dado que las razones para la implementación del cambio no han sido explicadas de forma adecuada y que el personal no fue incluido en el diseño del esquema de turnos.

La amenaza a los hábitos y la seguridad e intereses propios es otro de los factores de resistencia. Los entrevistados consideran que con la implementación del esquema de turnos propuesto, se afectarán sus rutinas familiares y sociales y, así mismo, se verán privados de ciertos beneficios con los que cuentan en el horario regular, tales como el servicio de alimentación.

En cuanto al diseño del plan de mitigación de los factores de resistencia al cambio se utilizaron sólo las estrategias de educación y comunicación, inclusión y participación, negociación y acuerdo propuestas por Kotter y Schlesinger (2008), puesto que se consideraron las más ade- cuadas ya que crean en el personal sentimientos de inclusión, compromiso con el éxito del cambio y acuerdos del tipo ganar-ganar. Se dejaron de lado las de facilitación y apoyo, manipulación y cooptación, y coerción. La primera de estas requería una inversión de tiempo y dinero muy elevada. La manipulación y cooptación, de ser descubiertas por el personal, crearian mayor resistencia y su inclusión tendría ciertos riesgos. Por último, no se utilizó la amenaza ya que el perfil del personal que labora en el departamento de base de datos es especializado y muy cotizado en el mercado laboral, por lo que sería probable su retiro de la Organización ante una situación de amenaza o chantaje. Además, por ser estrategias que pueden interpretarse como no éticas para el manejo de grupos humanos.

\section{Recomendaciones}

Realizar una evaluación que permita determinar si el plan diseñado contribuyó a disminuir la resistencia, para así tener certeza de la validez del mismo.

A la organización se recomienda, que ante la implementación de algún cambio que pueda tener impacto sobre la personas que allí laboran, las incluyan durante el diseño del mismo, para que desde un inicio se disminuya el impacto de los posibles factores de resistencia al cambio que se puedan presentar, ya que los individuos sentirán que son tomados en cuenta por la Organización y están participando de forma activa haciéndose responsables por el éxito del cambio y de la Organización.

El plan para la mitigación de los factores de resistencia al cambio, puede ser 
Resistencia al cambio y trabajo por turnos en trabajadores de la banca

Valero R., Daniel V. y Castilla Ramos, Orquídea

tomado como referencia por todas aquellas áreas de la Organización en las cuales se quiera implementar el esquema de turnos propuesto.

\section{Referencias bibliográficas}

Bennebroek, Kilian (2003). A different view on resistance to change. Symposium at the 11th EAWOP Conference. Power dynamics and organizational change IV. Lisboa, Portugal.

Bird, Robert (2007). A shift too far: The failure to recognize shiftwork maladaptation syndrome as an injury under workers' compensation law. Midwest Law Journal. En http://ssrn.com/abstract= 1057781, consultado el 19 febrero de 2009.

Böhrt, Raúl (2001) Resistencia al cambio y transición de las organizaciones. Tesis doctoral no publicada. Universidad Católica Boliviana, La Paz, Bolivia.

Buxton, Sandra (2003). Shift work: An occupational health and safety hazard. Tesis de Maestría. Murdoch University, Perth, Australia.

Carcelén, Javier (2000). El sistema de trabajo a turnos y su problemática. Madrid, España: Fundación Confemetal.

Congreso de la República de Venezuela (1997). Ley Orgánica del Trabajo. Gaceta Oficial No. 5.152 [Extraordinario], Venezuela.

Finn, Peter (1981). "Effects of shift work on employees". Monthly Labor Review, Vol. 104, No. 10, USA, U.S Department of Labor, pp. 31-35.

Folkard, Simón, Lombardi, David, y Tucker, Philip (2005). Shiftwork: Safety, sleepiness and sleep. Industrial Health, Vol. 43, No. 1, USA, NIOSH, pp. 20-23.
French, Wendell, y Bell, Cecil (1996). Desarrollo Organizacional. Aportaciones de las ciencias de la conducta para el mejoramiento de la organización (5ta ed.). Juárez, México, Prentice Hall, 375 pp.

García, Gustavo (2005). "Herramienta para el diagnóstico de la resistencia al cambio durante el desarrollo de proyectos mayores". Estudios Gerenciales, Vol. 21, No. 96, Colombia, Facultad de Ciencias Administrativas y Económicas, Universidad Icesi, pp. 57-106.

Hodge, Billy, Anthony, William y Gales, Lawrence (2003). Teoría de organización: Un enfoque estratégico. Madrid, España, Pearson Educación, $463 \mathrm{pp}$.

Kerlinger, Fred y Lee, Howard (2002). Investigación del comportamiento: Métodos de investigación en ciencias sociales (4ta ed). México, D.F: Mc Graw-Hill Interamericana, $810 \mathrm{pp}$.

Kotter, John y Schlesinger, Leonard (2008). Choosing strategies for change. Harvard Business Review, Vol. 86, No. 7, USA, Harvard Business School, pp. 130-139.

Medina, Yenny y Borgucci, Emmanuel (2005). Desempeño de la rentabilidad de los bancos fusionados en Venezuela entre los años 1998 y 2002. Revista Venezolana de Análisis de Coyuntura, Vol. XI, No. 1 (ene-jun), Venezuela, Instituto de Investigaciones Económicas y Sociales, Universidad Central de Venezuela, pp. 119-154.

Mellor, Earl (1986). How prevalent are shift work and flexitime? Monthly Labor Review, Vol. 109, No. 11, USA, U.S Department of Labor , pp. 14-21.

Nogareda, Clotilde y Nogareda, Silvia (1997). NTP 455: Trabajo a turnos y nocturno: Aspectos organizativos. Madrid, España: Instituto Nacional de Seguridad e Higiene en el Trabajo del 
Ministerio de Trabajo y Asuntos Sociales. En http://www.insht.es/lnsht Web/Contenidos/ Documentacion/FichasTecnicas/NTP/ Ficheros/401a 500/ntp_455.pdf, consultado el 11 de febrero de 2009

Nogareda, Silvia y Nogareda, Clotilde (1998). NTP 502: Trabajo a turnos: Criterios para su análisis. Madrid, España: Instituto Nacional de Seguridad e Higiene en el Trabajo del Ministerio de Trabajo y Asuntos Sociales. En http:// www.insht.es/InshtWeb/Contenidos/ Documentacion/FichasTecnicas/ NTP/Ficheros/501a600/ntp_502.pdf, consultado el 11 de febrero del 2009.

Occupational Health Clinics for Ontario Workers Inc. (2005). Shiftwork: Health Effects \& Solutions. Ontario, EE.UU.

Otero, María Carmen (2002). "Inercia Estructural: Antecedentes y Consecuencias". Revista Gallega de Economía, Vol. 11, No. 1. En http://www.usc.es/econo/RGE/Vol\%2011_1/ Castelan/Inercia $\% 20$ estructural. $\% 20$ Antecedentes $\% \quad 20 y \% 20$ consecuencias.pdf, consultado el 9 de febrero de 2009.

Pati, Atann Kumar, Chandrawanshi, Arti y Reinberg, Alain (2001). "Shift work: Consequences and management". Current Science, Vol. 81, No. 1, India, Current Science Association and Indian Academy of Sciences, pp. 32-52.
Robbins, Stephen (2004). Comportamiento Organizacional. México, D.F, Pearson Educación, 675 pp.

Rosa, Roger y Colligan, Michael (1997). Plain Language About Shiftwork. Cincinnati, Ohio, EE.UU, National Institute for Occupational Safety and Health.

Siegel, Sidney y Castellan, John (1995). Estadística no paramétrica (4ta ed.). México, D.F., México, Trillas.

Steinmetz, H. (2007). A multidimensional approach to working time. Tesis doctoral no publicada, Justus Liebig Universität Giessen, Alemania.

Suárez, Isabel (1999). El trabajo a turnos. Revista Bip, No. 19, pp. 50-53. En http:// www.ibermutuamur.es/IMG/ pdf/Ergonomia_bip19-2.pdf, consultado el 19 de febrero de 2009.

Szabla, David (2007). A multidimensional view of resistance to organizational change: Exploring cognitive, emotional, and intentional responses to planned change across perceived change leadership strategies. Human Resource Development Quarterly, Vol. 18, No. 4, USA, Wiley Periodicals, Inc., pp. 525-558.

Entrevistas:

García, Gustavo, Profesor UCAB; Caracas 3 de abril de 2009. 